\title{
Vícios e vicissitudes na adesão à terapêutica: um relato de caso
}

Sofia Lages Fernandes, ${ }^{*}$ Diogo Barata de Almeida, ${ }^{* *}$ Isabel Rebelo de Andrade, ${ }^{* * *}$ Márcia Gonçalves Lopes****

\section{RESUMO}

Introdução: A não adesão à terapêutica crónica é muito frequente, sobretudo no contexto da multimorbilidade. O objetivo da apresentação deste caso é sensibilizar para a aplicação do método clínico centrado no paciente (MCCP) como um instrumento eficaz para lidar com esta problemática.

Descrição do caso: Mulher, 49 anos, solteira, família unitária, com diagnósticos de tabagismo, diabetes mellitus tipo dois, hepatite $C$ crónica e com história prévia de consumo de heroína. Apresentava um mau controlo metabólico, que persistia após sofrer um AVC isquémico aos 48 anos, com parésia do membro superior esquerdo sequelar. Perante este contexto, convocou-se a utente para a realização de uma entrevista que englobava o estudo familiar, a exploração das crenças, expectativas, receios e importância atribuídos aos seus problemas de saúde, bem como a revisão dos hábitos e adesão à terapêutica.

Apurou-se não haver evidência de disfunção familiar. Ao rever os hábitos identificou-se uma compulsão para doces, consumo atual de haxixe e tabaco, que eram desvalorizados pela própria enquanto problemas de saúde. Confirmou-se a suspeita de incumprimento da posologia de cinco dos oito fármacos prescritos, por desconhecimento da razão da sua prescrição e por receio de efeitos secundários.

Como plano mobilizou-se a equipa de cuidados de saúde primários e secundários com vista a reavaliar a terapêutica não farmacológica e farmacológica, de acordo com as necessidades atuais da utente.

Nas consultas subsequentes verificou-se total adesão à medicação e autocontrolo glicémico e parcial adesão à dieta. Constatou-se então um melhor controlo metabólico (HgA1c passou de 10,7\% para 7,8\%).

Discussão: Este é um caso de multimorbilidade e de não adesão à terapêutica. Para abordá-lo é necessária multidisciplinaridade, uma relação médico-doente e comunicação eficazes. Nesse sentido, o MCCP é um instrumento essencial e o médico de família é o especialista privilegiado para o aplicar.

Palavras-chave: Multimorbilidade; Adesão à Terapêutica; Método Clínico Centrado no Paciente.

\section{INTRODUÇÃO}

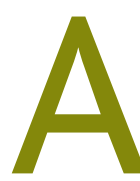
não adesão à terapêutica crónica nos países desenvolvidos é estimada em $50 \%{ }^{1-2}$ Esta pode causar complicações médicas e psicossociais, reduzir a qualidade de vida e desperdiçar recursos de cuidados de saúde. ${ }^{1}$ Acresce ainda a sua dificuldade diagnóstica, sendo necessário o profissional de saúde estar sensibilizado para esta problemática. Quanto à sua abordagem, segundo uma revisão da Cochrane

* Médica interna de Medicina Geral e Familiar na USF Descobertas **Médico interno de Medicina Geral e Familiar na USF Descobertas

***Médica assistente graduada de Medicina Geral e Familiar na USF Descobertas

****Médica assistente graduada de Medicina Geral e Familiar na USF Descobertas de 2014, a evidência atual é insuficiente para recomendar intervenções significativamente eficazes. ${ }^{2}$

O presente caso clínico retrata uma mulher de 49 anos com multimorbilidade e com complicações graves das suas patologias. Por esse motivo, a utente foi convocada para realização do estudo familiar, para conhecer melhor a sua perspetiva sobre os seus problemas de saúde, para revisão dos seus hábitos e avaliação da adesão à terapêutica não farmacológica e farmacológica. Esta abordagem baseou-se na aplicação do método clínico centrado no paciente (MCCP). ${ }^{3}$

Pretende-se, assim, alertar para a importância da identificação e contextualização da não adesão à tera- 
pêutica, de forma a abordá-la eficazmente através da aplicação do MCCP.

\section{DESCRIÇÃO DO CASO}

\section{Identificação}

Isabel (nome fictício), sexo feminino, 49 anos, caucasiana, natural e residente no concelho de Lisboa, com mestrado na área da educação, à data da realização da entrevista encontrava-se desempregada. Tem, como ocupações preferidas, ler e ouvir música.

A utente constitui uma família unitária, é solteira, pertence à classe social média-alta (Graffar Familiar de classe social grau II) e é independente para as atividades de vida diária básicas e instrumentais.

\section{Contexto clínico}

Isabel iniciou o seu seguimento na atual médica de família em outubro de 2013 e, nessa data, eram conhecidos, como problemas de saúde ativos, o tabagismo desde os 11 anos de idade, hepatite $\mathrm{C}$ diagnosticada aos 26 anos e diabetes mellitus tipo 2 aos 33 anos e, como problema de saúde passivo, o abuso de drogas com consumo de heroína até aos 38 anos de idade.

Revelava persistentemente um mau controlo metabólico (Quadro I), grande dificuldade em alterar os hábitos alimentares, em efetuar autocontrolo glicémico e recusava ser encaminhada para tratamento da hepatite C. Revelava-se uma pessoa assídua nas consultas, diferenciada, com conhecimento científico e cultural significativo.

Em maio de 2014 sofreu um acidente vascular cerebral (AVC) isquémico direito no contexto de estenose da artéria carótida interna direita com $75-80 \%$ de obstrução, sendo submetida a angioplastia. Após alta hospitalar apresentava, como sequela, parésia do membro superior esquerdo e ficou com seguimento em consulta hospitalar de medicina física e reabilitação, neurologia, cirurgia vascular, endocrinologia e de infecciologia.

Após alta revelou uma melhoria inicial do controlo metabólico, mas apenas transitória (Quadro I), voltando a não aderir às medidas terapêuticas propostas com consequente mau controlo metabólico. Perante este contexto, convocou-se a utente para uma entrevista com o objetivo de tentar compreendê-la melhor no seu
QUADRO I. Lista de resultados de hemoglobinas glicadas

\begin{tabular}{l|c|l} 
Data & $\begin{array}{c}\text { Hemoglobina } \\
\text { glicada A1C }\end{array}$ & \\
\hline $8 / 12 / 2013$ & $10,6 \%$ & \\
$28 / 03 / 2014$ & $10,2 \%$ & \\
$9 / 05 / 2014$ & $10,3 \%$ & AVC isquémico a $16 / 05 / 2014$ \\
$2 / 09 / 2014$ & $8,3 \%$ & \\
$02 / 12 / 2014$ & $9,1 \%$ & \\
$09 / 06 / 2015$ & $10,7 \%$ & Intervenção do MF em maio de 2015 \\
$0 / 10 / 2015$ & $7,8 \%$ &
\end{tabular}

todo e de ajudá-la a melhorar o seu estado de saúde de forma mais eficaz.

\section{Aplicação do método clínico centrado no paciente (MCCP) na entrevista}

Na operacionalização do MCCP devem ser consideradas seis componentes: exploração da doença e da dolência; compreensão da pessoa no seu todo e no seu contexto; estabelecimento de um terreno comum; promoção da saúde e prevenção da doença; construção da relação médico-doente e ser-se realista. ${ }^{4}$

A aplicação do MCCP deve decorrer de forma fluída, de acordo com as pistas verbais e não verbais que $o$ utente dá ao longo da entrevista, tendo como base a escuta ativa. ${ }^{5}$

Sem prejudicar este princípio, procurou-se conhecer melhor a utente, a sua história de vida e familiar, bem como as suas ideias, crenças, expectativas e receios sobre os seus problemas de saúde. Assim, foi possível rever os seus hábitos, conhecer o seu estilo de vida, as suas motivações e preocupações, bem como a adesão à terapêutica não farmacológica e farmacológica.

Como resultado, o conhecimento aprofundado da agenda da utente permitiu estabelecer um terreno comum na identificação dos problemas de saúde e na negociação do plano terapêutico.

\section{Compreensão da pessoa no seu todo e no seu con- texto}

No genograma (Figura 1) destacou-se que Isabel viveu em união de facto durante seis anos, até 1997, e que atualmente vive sozinha. Tem uma irmã onze anos mais 


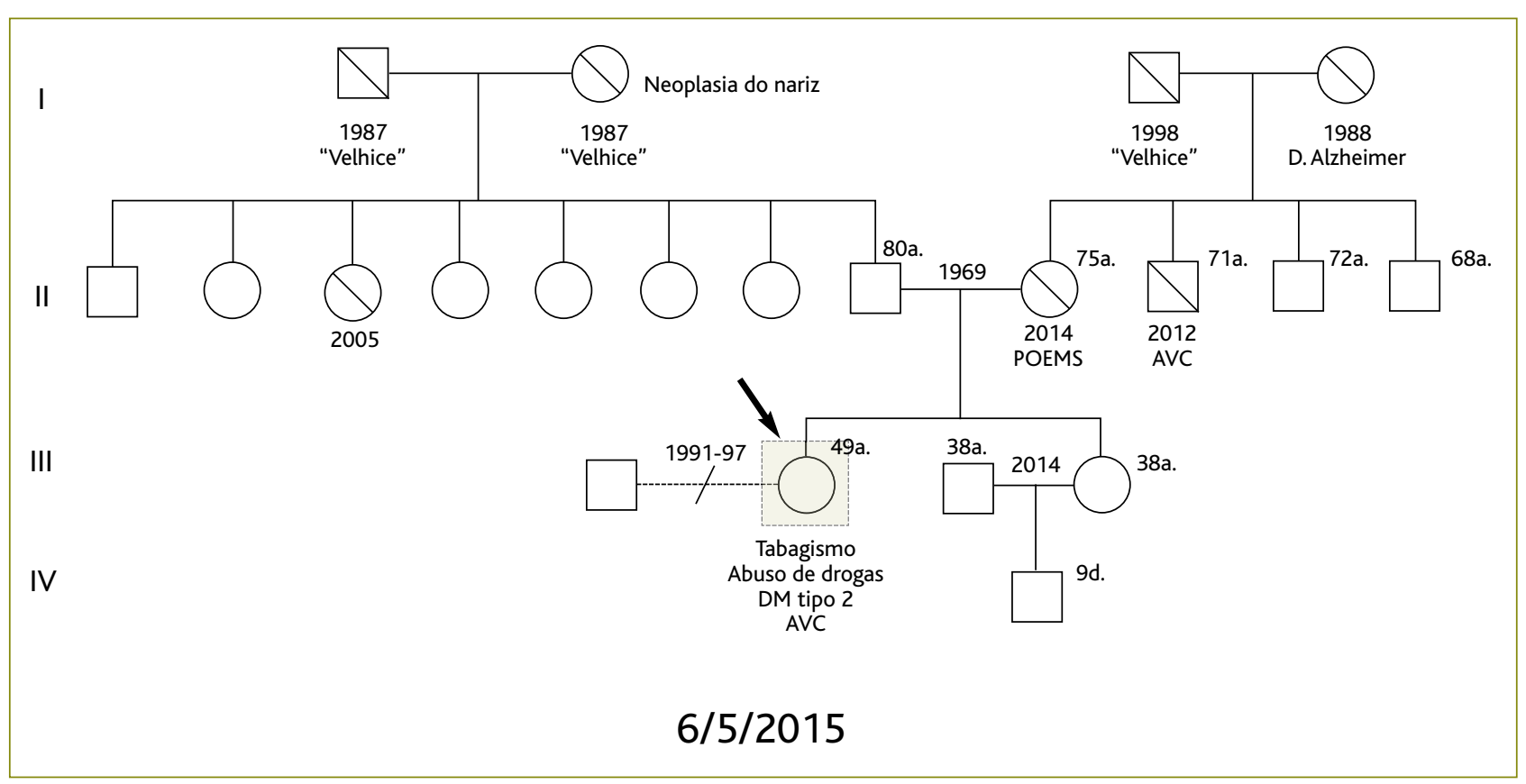

Figura 1. Genograma realizado a 06/05/2015.

nova, casada, que teve um filho recentemente. Salientava-se ainda que a mãe falecera em 2014 por uma doença neurológica - polineuropatia periférica sensitivo-motora desmielinizante crónica (síndroma POEMS). Não se verificou um padrão familiar de dependências, de fatores de risco ou de patologias.

Relativamente à psicofigura de Mitchell (Figura 2), Isabel revelava ter uma ótima relação com os pais. Contudo, sentia que o pai se distanciava mais dela e via a sua toxicodependência como uma escolha, por oposição à mãe, que se mostrava mais próxima e considerava-a como uma doença. Quanto à relação entre os pais e a irmã, revelou que "a minha irmã sempre foi a filha que os pais querem ter", em tom factual, sem índice de ciúme. Isabel considerou ter uma relação muito próxima com a irmã, que se preocupava muito com ela, assumindo "a minha irmã é mais nova onze anos que eu mas tem sido como minha mãe". Quando se abordou a relação com o cunhado, revelou não gostar dele ("Não me faz nada de mal, mas irrita-me"). Quando questionada sobre a causa da sua irritação, confessou que talvez sentisse ciúmes dele porque lhe "roubou" a irmã que agora tinha de despender mais tempo com a sua nova família.
Ao realizar o círculo familiar de Thrower (Figura 3), Isabel mostrou-se satisfeita com a representação que fez e quando se questionou sobre a quem recorreria, caso precisasse de ajuda, referiu a irmã e o pai. Após assinalar-se a ausência do cunhado na representação, Isabel referiu que o fez propositadamente pois não o considerava parte da sua família.

Ao realizar a biopatografia (Quadro II), destacou-se:

- Início do tabagismo aos 11 anos, coincidente com o nascimento da irmã.

- Início do consumo de haxixe aos 14 anos como "símbolo de rebeldia, por influência social, sem nenhuma consequência negativa".

- Início do consumo de heroína aos 18 anos, porque "surgiu este novo produto quando havia crise nacional de haxixe e desconhecia as consequências...".

- Inconstância académica e ocupacional.

- Relato de uma única relação amorosa duradoura: aos 25 anos emigrou para Espanha na tentativa de abstinência dos consumos toxicofílicos. Contudo, agravou-os e iniciou relação com um toxicodependente com quem viveu em união de facto durante seis anos, chegando com o mesmo a "viver na rua". "Depois de ter dado um mergulho muito maior no 
mundo da heroína" e por carência económica decidiu terminar a relação amorosa e voltar para Portugal para tentar deixar a heroína, uma vez que o companheiro queria continuar.

- Viveu com os pais e teve várias tentativas para deixar a droga, tendo conseguido, por fim, em 2004, com apoio da médica de família. Referiu que a deceção causada na família foi a sua principal motivação, assim como a vontade de deixar de viver para uma dependência.

- Concomitância entre problemas de saúde e acontecimentos vitais: diagnóstico de infeção por vírus da hepatite $C$ três anos depois de iniciar consumo de heroína; AVC isquémico no ano da morte da mãe e do casamento da irmã.

\section{Revisão dos hábitos}

Hábitos tabágicos: Fuma desde os onze anos de idade, carga tabágica de 37,5 unidades maço ano. Único período de interrupção: durante internamento por AVC isquémico.

Hábitos alcoólicos: Consumo social (quinzenalmente), em cada um dos eventos sociais bebe 58g álcool. Aplicou-se o questionário AUDIT, cujo resultado foi de 4 pontos.

Hábitos toxicofílicos: Aos 14 anos iniciou consumo de haxixe que mantém atualmente em eventos sociais (cerca de uma vez por mês). Aos 18 anos iniciou consumo de heroína inalada e, posteriormente, injetável até aos 38 anos de idade. Consumiu também LSD, cocaína e metanfetaminas, estas até há cerca de três anos.

Hábitos alimentares: Isabel descreveu a sua alimentação num dia tipo e apurou-se que ingeria quatro refeições por dia e que consumia diariamente cerca de seis porções de hidratos de carbono, excluindo açucares, seis peças de fruta, duas vezes laticínios, duas a três vezes vegetais e em média dois a três doces por dia. Ingeria peixe ou carne em média em cinco refeições numa semana.

Hábitos de exercício físico: Não praticava.

\section{Exploração da doença e da dolência}

Quando se questionou qual o problema de saúde que tinha maior importância para a utente, esta referiu ser a parésia do membro superior esquerdo, uma vez que tinha repercussão no seu dia-a-dia. Apesar de con-

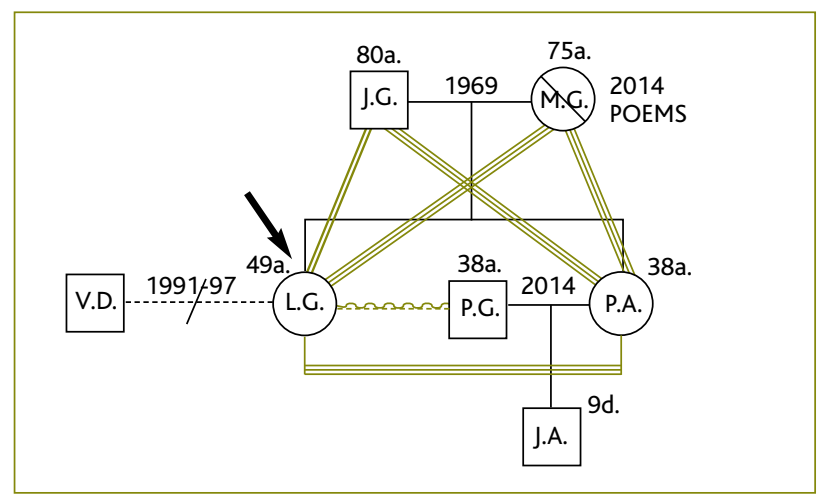

Figura 2. Psicofigura de Mitchell realizada a 06/05/2015.

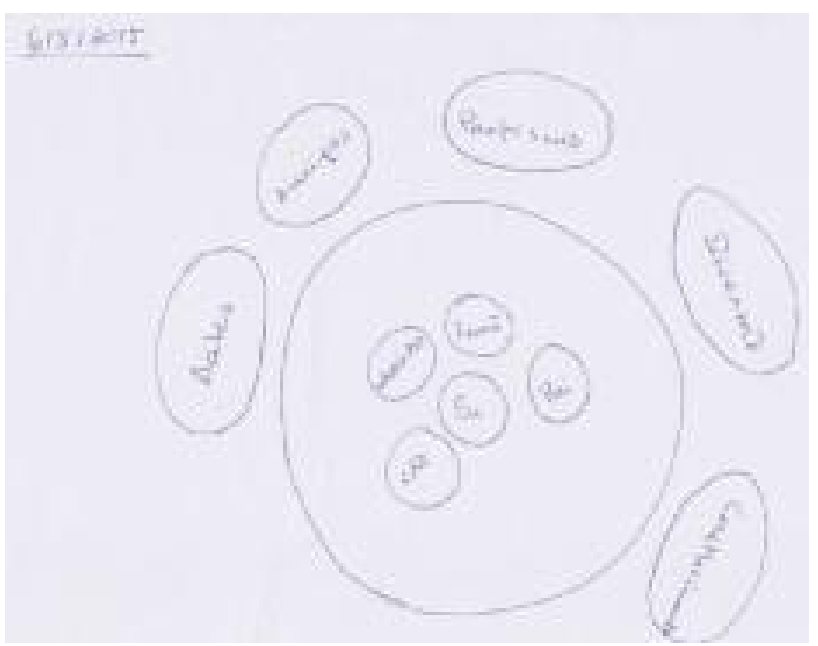

Figura 3. Círculo familiar de Thrower.

seguir executar todas as atividades de vida diária de forma autónoma, referiu uma maior lentidão e dificuldade em realizar algumas dessas atividades. Contudo, mostrou-se otimista, pois tem notado uma melhoria significativa com o apoio da medicina física e reabilitação.

A segunda preocupação manifestada pela utente foi a possibilidade de sofrer novo AVC. Isabel atribuía como possível causa para o AVC prévio uma queda que tinha tido há quatro meses, desvalorizando os fatores de risco vasculares na sua etiologia, nomeadamente o tabagismo. Efetivamente, assumiu que não estava motivada para deixar de fumar, pois "podia ser fator de risco mas não [lhe garantiam] que não [tinha] novo AVC se deixasse de fumar". 


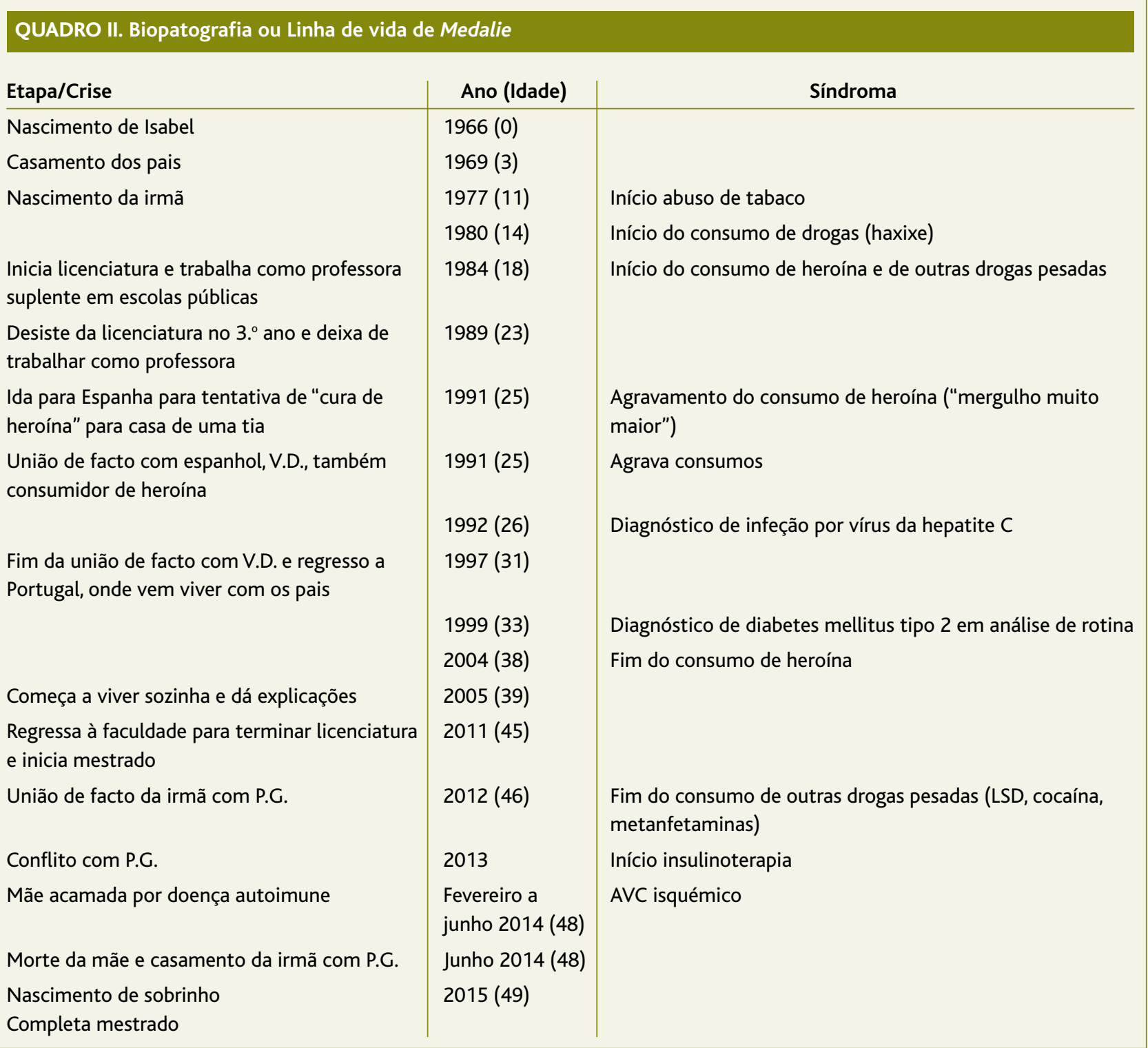

Em terceiro lugar, a utente considerou a diabetes como uma das suas maiores preocupações pois "tem sido uma complicação". Revelou sofrimento porque, por um lado, sentia-se incapaz de controlar o consumo de doces, classificando-o como uma dependência pior que a droga ("a droga é mais fácil de lidar do que os doces porque não há montras de drogas"). Por outro lado, revelou dificuldade em efetuar a insulinoterapia, nomeadamente no que respeita à insulina rápida, à qual atribuía aumento ponderal. Manifestou ainda relutân- cia em vigiar a glicémia capilar, por "não gostar de picadas" que a remetiam para o seu passado de consumo de heroína.

De seguida, Isabel revelou recear os possíveis efeitos secundários da terapêutica para a hepatite $\mathrm{C}$, por relatos de amigos, valorizando-os mais do que a própria doença. Esta nunca lhe causou queixas ou sofrimento, pelo que para si não era um problema.

De salientar ainda que a utente não considerou o consumo atual de haxixe como um problema de saú- 
de, declarando "chamar-lhe abuso [de drogas] é abusar". Explicitou que "para um toxicodependente o haxixe não é uma droga" e manifestou desconhecimento sobre os riscos do seu consumo. Pelo contrário, referiu vários benefícios, como a melhoria da concentração e da capacidade de trabalho, negando qualquer receio.

\section{Revisão da adesão à terapêutica farmacológica}

À data da primeira sessão da convocatória apurou-se a perspetiva da utente em relação à terapêutica farmacológica prescrita e respetiva adesão:

- Omeprazol 20mg/l comprimido em jejum - Tomava diariamente "porque é protetor para o estômago".

- Ramipril 5mg/1 comprimido ao pequeno-almoçoPor auto iniciativa, no último mês tomava em dias alternados por desconhecer o seu motivo ("nunca tive problemas de tensão, achei um exagero darem este medicamento desde o meu internamento").

- Carbamazepina $200 \mathrm{mg} / 1$ comprimido ao pequeno-almoço e ao jantar - Apurou-se que apenas tomava um comprimido diário por desconhecer o seu motivo ("não sou epilética").

- Clopidogrel 75mg/1 comprimido ao almoço - Tomava diariamente.

- Ácido acetilsalicílico 150mg/l comprimido ao almoço-Tomava apenas em dias alternados por receio de hemorragias.

- Atorvastatina $40 \mathrm{mg} / 1$ comprimido ao deitar - Tomava em dias alternados por desconhecer a razão da sua prescrição ("nunca tive problemas de colesterol").
- Insulina glargina 30 UI ao deitar e insulina rápida aspártico 3UI+3UI+3UI - Fazia a insulina glargina, mas recusava-se a fazer insulina rápida porque referia aumentar de peso.

Concluindo, à data do início do relato, a utente recusava tomar um dos oito fármacos prescritos, cumpria a posologia prescrita apenas em três deles e tomava os restantes de acordo com as suas crenças e expectativas.

\section{PLANO}

Confirmou-se a suspeita de não adesão às medidas não farmacológicas e farmacológicas prescritas. Tendo em conta os problemas ativos e passivos atualizados da utente (Quadro III), foram planeadas as seguintes estratégias não farmacológicas em conjunto com a utente e a enfermeira de família:

- Alimentação: negociou-se um novo plano alimentar que visava sobretudo o polifracionamento das refeições ao longo do dia e a redução da ingesta de fruta e de doces.

- Exercício físico: a utente não demonstrou interesse na prática desportiva, mas sugeriu aumentar a utilização de transportes públicos e caminhar mais vezes a pé, não determinando uma duração mínima para as caminhadas.

- Hábitos nocivos: uma vez que Isabel estava em fase pré-contemplativa no ciclo de mudança para a cessação tabágica e consumo de haxixe, exploraram-se os riscos em manter estes consumos, bem como a relevância dos benefícios, as recompensas e as resis-

\section{QUADRO III. Lista de problemas ativos e passivos}

\section{Problemas Ativos}

Doença cérebro-vascular

Parésia MSE desde há 2 anos

DM tipo 2 diagnosticada há 16 anos

Hepatite $C$ crónica diagnosticada há 23 anos

Consumo de haxixe há 35 anos

Tabagismo há 38 anos
Problemas Passivos

AVC Isquémico ACM direita (05-2014) com colocação stent $\mathrm{ACl}$ por estenose pré-oclusiva (06-2014)

Consumo de heroína (1984-2004)

Consumo de outras drogas pesadas (1984-2012)

Legenda: ACl - Artéria Carótida Interna; MSE - Membro Superior Esquerdo; DM - Diabetes Mellitus; ACM - Artéria Cerebral Média. 
tências em cessá-los. A utente não manifestou vontade de alterar os seus hábitos, pelo que se planeou a repetição desta intervenção breve dos cinco "Rs" em próximas consultas.

- Auto controlo da glicémia capilar: foram revistos os ensinos sobre a medição da glicémia capilar, valoresalvo, bem como o reconhecimento dos sintomas de hipoglicemia e respetiva abordagem adequada. A utente concordou iniciar o registo de glicémias.

Relativamente à terapêutica farmacológica foram esclarecidas as dúvidas e crenças identificadas sobre a medicação, nomeadamente: foram explicadas as razões da prescrição da estatina (reforçando a sua ação antiaterogénica e consequente redução da probabilidade de ter novo AVC); da dupla antiagregação (para evitar a trombose do stent vascular) e da insulinoterapia (importância deste fármaco para melhorar o controlo metabólico e reduzir as complicações associadas à diabetes). Isabel demonstrou ter compreendido e pareceu aceitar estes esclarecimentos.

Também foram tidos em conta os receios e expectativas da utente relativamente à insulinoterapia, pelo que se ponderou acrescentar antidiabéticos orais com ação neutra ou negativa no peso, que a utente se mostrou recetiva a tomar. Foi, então, enviada carta ao endocrinologista onde se expuseram, com o consentimento da utente, os seus receios e incumprimento terapêutico e foi sugerida a alteração mencionada. O endocrinologista iniciou então uma associação de metformina com vildagliptina e alterou o esquema de insulinoterapia para o uso de uma insulina pré-mistura duas vezes por dia. A utente expressou agrado perante estas alterações da terapêutica.

Quanto ao ramipril e à carbamazepina, uma vez que a utente não tinha história pessoal ou familiar de hipertensão ou de epilepsia, enviou-se carta à neurologista questionando a razão da toma destes fármacos e pedindo informação acerca de eventuais diagnósticos desconhecidos de hipertensão ou epilepsia. Como resposta, a neurologista suspendeu o ramipril e, progressivamente, a carbamazepina e deu alta para a médica de família, esclarecendo que a prescrição tinha sido realizada por a utente ter tido crise hipertensiva e crise epilética sintomática aguda no contexto de instalação de lesão cerebral aguda. Mais uma vez, estas alterações foram de encontro às expectativas da utente.
Promoveu-se, ainda, o esclarecimento sobre a hepatite $\mathrm{C}$ e respetivas complicações e tratamento, desmistificando os receios sobre os seus efeitos secundários.

\section{SEGUIMENTO}

No seguimento da utente, cinco meses depois, a mesma refere que tem tomado todos os medicamentos, incluindo a insulina e que tem efetuado o autocontrolo glicémico. Refere que tem ainda muita dificuldade em melhorar os hábitos alimentares, sobretudo na redução da ingesta de fruta e doces, apesar de fazer um maior número de refeições (cerca de cinco por dia). Objetivamente apura-se uma redução significativa do valor de hemoglobina glicada (em que a 10.10.2015 era de $7,8 \%$ ), sem se apurar hipoglicémias.

A utente tem cumprido as consultas de seguimento de infecciologia. Numa das consultas subsequentes de medicina geral e familiar, Isabel expressa vontade em tratar a hepatite C e preocupação pelo seu estado de saúde, aguardando chamada para início de terapêutica a nível hospitalar.

A utente revela ter conseguido emprego como professora a tempo inteiro. No âmbito familiar, não voltou a ter conflitos com o cunhado. A irmã da utente nota também melhoria do compromisso de Isabel com a sua saúde e mostra-se muito satisfeita e grata aos prestadores dos cuidados de saúde.

Como desafios mantém-se a promoção da cessação tabágica e do consumo de drogas, bem como a manutenção da adesão às medidas negociadas.

\section{COMENTÁRIO}

Este caso ilustra duas situações complexas e comuns na prática clínica: a não adesão à terapêutica e a multimorbilidade.

Um fator determinante para a adesão terapêutica é uma relação paciente-médico eficaz que permita explorar alternativas terapêuticas, negociar o plano, discutir a adesão e planear o follow-up. ${ }^{1} \mathrm{O}$ desenvolvimento da relação paciente-médico é a pedra basilar do $\mathrm{MCCP}^{4}$ que, por sua vez, conduz a uma maior adesão aos tratamentos. ${ }^{5}$ Para concretizar a aplicação deste método é necessário utilizar uma comunicação efetiva que englobe escutar, falar e compreender. ${ }^{4}$

Neste caso, a compreensão do contexto familiar e 
história de vida da utente bem como das suas crenças, receios e expectativas permitiu reforçar a relação e esclarecer a agenda da utente. Esta abordagem levou a uma comunicação mais eficaz, ao estabelecimento de um terreno comum sobre os problemas de saúde e à negociação do plano terapêutico.

Existe ainda evidência de que muitos pacientes com doenças crónicas, incluindo a diabetes, têm dificuldade em aderir aos regimes terapêuticos ${ }^{1}$ e que o MCCP é essencial quando se considera que um sistema de saúde deve cuidar de pessoas com multimorbilidade. ${ }^{6-}$ ${ }^{7}$ Por outro lado, os pacientes com multimorbilidade estão ligados a vários médicos e serviços, não coordenados entre si e ficam confusos quanto a quem é responsável e por quê, podendo existir problemas insuficientemente controlados e outros duplamente tratados. $^{7-8}$ Infere-se que a multimorbilidade exige multidisciplinaridade, colocando-se a questão de como é que o médico de família pode concretizar o seu papel enquanto gestor de cuidados.

Neste caso optou-se por comunicar por escrito com os colegas as dificuldades e sugestões, de acordo com as preocupações da utente. A multidisciplinaridade deve, então, refletir um esforço coordenado por parte dos diferentes profissionais de saúde com o objetivo comum de melhorar a saúde da pessoa, ao invés de reduzir-se ao tratamento de uma dada doença. ${ }^{6-7}$

A aplicação do método clínico centrado no paciente é essencial para fomentar a adesão à terapêutica crónica e para cuidar de pessoas com multimorbilidade., ${ }^{3,5-6}$
REFERÊNCIAS BIBLIOGRÁFICAS

1. World Health Organization. Adherence to long-term therapies: evidence for action [Internet]. Geneva:WHO; 2003. ISBN 9241545992. Available from: http://apps.who.int/iris/bitstream/10665/42682/1/ 9241545992.pdf

2. Nieuwlaat R, Wilczynski N, Navarro T, Hobson N, Jeffery R, Keepanasseril $A$, et al. Interventions for enhancing medication adherence. Cochrane Database Syst Rev. 2014;(11):CD000011.pub4.

3. Stewart M, Brown J, Levenstein J, McCracken E, McWhinney IR. The patient-centred clinical method. 3. Changes in residents' performance over two months of training. Fam Pract. 1986;3(3):164-7.

4. Nunes JM. Comunicação em contexto clínico. Lisboa: Bayer; 2010.

5. McWhinney IR, Freeman T. Manual de medicina de família e comunidade. $3^{\text {a }}$ ed. Porto Alegre: Artmed; 2010. ISBN 9788536321257

6. Boyd CM, Fortin M. Future of multimorbidity research: how should understanding of multimorbidity inform health system design? Public Health Rev. 2011;33(2):451-74.

7. Broeiro P. Multimorbilidade e comorbilidade: duas perspetivas da mesma realidade [Multimorbidity and comorbidity: two perspectives on the same reality]. Rev Port Med Geral Fam. 2015;31(3):158-60. Portuguese

8. Doessing A, Burau V. Care coordination of multimorbidity: a scoping study. J Comorbidity. 2015;5(1):15-28.

\section{CONFLITO DE INTERESSES}

Os autores declaram não ter conflitos de interesses.

\section{ENDEREÇO PARA CORRESPONDÊNCIA}

Sofia Lages Fernandes

USF Descobertas, ACES Lisboa Ocidental e Oeiras, ARS Lisboa e Vale do Tejo E-mail: sofia.f3@gmail.com

Recebido em 17-04-2015

Aceite para publicação em 18-11-2016 


\section{ABSTRACT}

\section{ADDICTIONS AND OTHER OBSTACLES TO ADHERENCE TO THERAPY:A CASE REPORT}

Introduction: Non-adherence to therapy is a prevalent problem, especially among patients with multiple comorbidities. The aim of this case report is to raise awareness of the importance of the patient-centered clinical method in dealing with this issue. Case report: We present the case of a 49-year-old single female living alone with type 2 diabetes, hepatitis $C$ infection, and a previous history of drug abuse. She had poor metabolic control even after suffering an ischemic stroke at 48 years of age, which left her with weakness of her left arm. The patient was invited by her family physician for a family assessment interview, including a review of her habits and medications and an exploration of her beliefs, fears, and expectations about her health problems and their importance to her.

There was no evidence of family dysfunction but she was found to have a compulsion to eat sweets and she reported current hashish and tobacco consumption. Her poor adherence to therapy was confirmed. She was only taking three out of eight medications prescribed, due to a lack of awareness of their indications and fear of adverse effects.

The patient's ideas and fears about her treatment were explored and clarified, and therapy was optimized according to her expectations. Nursing support was obtained and contact was made with her neurologist and endocrinologist.

In subsequent visits, adherence to therapy reported by the patient was improved along with partial adherence to the recommended diet, resulting in improved metabolic control.

Discussion: This is a case report of a patient with multimorbidity and non-adherence to therapy. The patient benefitted from a multidisciplinary approach, a good doctor-patient relationship, and effective communication. The patient centered clinical method was helpful in achieving these goals and the family physician was well placed to apply it.

Keywords: Multimorbidity; Adherence to Therapy; Patient-centered Clinical Method. 